the other one is "Felis -... 14. Femur Right side." There is no record in the catalogue by whom they were presented, nor of any of the circumstances of their gisement. The specimens, in fact, have no history whatever, and I can only say that I found then in close juxtaposition with a large series of red-deer bones from Iolderness, with which they perfectly agree in their mineralogical condition. I have no doubt that they are bon $\hat{a}$ fide from the Holderness Peat.

Their identification as bones of $F$. leo (variety spelara) is also certain.

Hull Royal Institution

$$
\text { C. Cartror Blake }
$$

\section{Eozoön Canadense}

SiNCE reading some of the communications on the Eozoön, which have appeared from time to time in NATURE, I have felt constrained briefly to give the results of my examination of the "Eozoic" limestone in Eastern Massachusetts. I am the more disposed to do this, hoping that a new line of investigation will be suggested to observers in other localities.

Last autumn I visited for the first time the quarries of "Eozoön" limestone in Chelmsford, under the guidance of my friend Mr. Burbank, of Lowell, Massachusetts, who has furnished many microscopists with specimens for sections. Having been long engaged in the study of the foliated series of rocks, and having years ago discovered indubitable evidence that portions of the included limestone are of vaporous origin, I was prepared to recognise the same feature in the Chelmsford "Eozoic" rock. I was accordingly not surprised on examination to find, what the advocates of the organic nature of the Eozoon seem never to have suspected, that the limestone in question is not a "sedimentary rock;" that it occupies, or rather occupied, (for it has been for the most part removed) pockets or ovenshaped cavities, which were once plainly overarched by gneiss; that it is foliated, there being a regular succession of leaf-like layers from the walls toward the centres of the cavities, witness to which is borne by a like succession of different minerals; that in some places it ramifies the surrounding rock in a vein-like way, while in others it exactly conforms with the most abrupt irregularities of surface; that in one locality, which I have repeatedly examined, it conforms with the uneven portions of a mass of syenite, with which it is so associated as to reveal its more recent origin; and that, therefore, it is not of nummulitic derivation, but was deposited in a vein-like form, the materials having been probably forced up into the cavities from below while in a vaporous state.

Such, in few words, is the result of my examination-a result which tends to show that the "Eozoön" of Eastern Massachusets is not organic, and that thus it belongs to the department of Mineralogy, and not to that of Palæontology. Waving additional particulars for the present, I may simply add that I propose in due time to give a detailed exposition of the relations of this famous "Eozoic" rock.

Cambridge, Mass., A pril 15

JOHN B. PERRY

\section{THICKNESS OF THE EARTH'S CRUST}

I SEE that at p. 296 of your journal for February last, which has recently reached Calcutta, you print a lecture by Mr. David Forbes "On the Nature of the Earth's Interior," in which reference is made to the Mr. W. Hopkins's method of determining whether the thickness of the earth's crust is great or small when compared with the whole radius, and to M. Delaunay's objection to it.

The lecturer refers to me as having approved of $\mathrm{Mr}$. Hopkins's method, which I always have done and do still, and then makes the following apparently crushing remarks to annihilate Mr. Hopkins and all who approve of his method and of the result to which it leads, viz., that the crust is very thick. He says:- "M. Delaunay, an authority equally eminent as a mathematician and an astronomer, was induced to undertake the reconsideration of the problem; a labour (!) which has resulted in altogether reversing the above decision and demonstrating the complete fallacy of the premises upon which so much elaborate reasoning had been expended."
As the lecturer had condescended to mention my name in connection with the subject, I wonder why he has taken no notice of my letter in reply to $M$. Delaunay, which was printed in your journal for July 1870 , six months before the lecture was delivered, and which also appeared about the same time in the Philosophical Magazine and the Geological Magazine. In this I showed that M. Delaunay had evidently misconceived the problem, and that $\mathrm{Mr}$. Hopkins's method is altogether unaffected by his remarks.

So much has been said about profound mathematical calculations in connection with Mr. Hopkins's investigation, that I conceive many have shrunk from attempting to understand the question at issue, from a feeling that they would not be able to comprehend it were they to attempt to do so. But this is quite a mistake. Anyone with an ordinary degree of knowledge of popular astronomy and of mechanical action is quite competent to form a good opinion on the point in dispute. What Mr. Hopkins did may be divided into two parts. He first conceived an idea, which was to be the basis of his calculation; and then he made his calculation. It is the calcut?ation that calls for the "profound mathematics." But it is not this that is the matter of dispute. It is the idea, on which the calculation is based, which M. Delaunay calls in question.

I think I can make the matter sufficiently plain to your readers to enable them to form their own opinion.

Everyone having a knowledge of popular astronomy is aware that the earth revolves round an axis, which is fixed in the earth's solid crust, but shifts very slowly in space, producing what has been known ever since the days of Hipparchus by tine name Precession. On this fact as his ground-work Mr. Hopkins reasoned as follows; and so got to his idea, which formed the basis of his calculation. Suppose the earth has a solid crust, the interior being filled up with fluid. If the axis remained steady in space and the crust revolved round it uniformly, no doubt, although the crust and fluid may have moved differently at one time, yet in the lapse of ages friction and viscosity in the fluid would cause the fluid at last to revolve with the crust just as if the whole were one solid mass. This being the case, suppose a slight horizontal push is given to the two poles, in opposite directions, so as slightly to shift the axis in space; what would happen? The revolving crust, by this new and additional motion, would slip over the surface of the rivolving fluid, through a small space proportionate to the push given to the poles. The fluid could not possibly acquire in an instant this new motion, however small it might be, because the fluid is not rigidly connected with the crust. Suppose a second, and a third, and a succession of slight horizontal pushes to be given to the poles in a continually altering direction, the effect will be that the revolving crust will be continually slipping over the revolving fluid which has not time to acquire these new motions given instantaneously to the solid crust. These successive slight pushes given to the poles, and so to the solid crust, represent the unceasing action upon the crust of the force which causes the motion of precession in the earth's axis, and arises from the attraction of the sun and moon on the protuberant parts of the earth about the equator.

Mr. Hopkins having reasoned thus far, went a step farther, and so came to his fundamental idea. He saw that the thinner the crust the smaller would be the mass which the disturbing force producing precession would have to move, and therefore the greater would be the motion caused, that is, the precession. Here, then, he discerned a connecting link between the amount of precession of the earth's axis and the thickness of the earth's crust. This was the idea I have aliuded to.

Starting from this idea he entered upon a profound calculation and obtained a formula, which gives the thickness in terms of the amount of precession. This amount is a matter of observation; and the thickness can therefore be deduced by the formula from the observed pre- 
cession. It is, as I have already said, not this calcula. tion which is called in question by $\mathbb{M}$. Delaunay, but the fundamental idea.

M. Delaunay says the fluid will have precisely the same motion as the crust; and that, because the new motion of the crust is so slow. But it is clear that its s?owness has nothing to do with the matter. The fact is that the fluid and the crust not being connected together by any solid connection, no motion, whether small (i.e. slow) or not, can be suddenly communicated from the crust to the fluid mass. If the crust moved uniformly, as I have already said, and around a steady axis, the fluid might, after a lapse of ages, by friction and viscosity, acquire the motion of the crust. But if the crust is continually shifting from this steady position, however slowly, the fluid cannot suddenly acquire the new motion, and the crust slips over it ; and the thicker or thinner the crust, the greater or less is the solid mass to be shifted, and the less or the greater the precession produced, If the internal mass obeys at once the shifting motions of the crust, that mass cannot be fluid, but must be solid, and have a solid connection with the crust; in which case the whole question is yielded.

Mr. David Forbes speaks of the "Iabour" M. Delaunay has gone through in giving vent to his opinion. If the thing done is to be measured at all by the thing said, his labour must have been infinite; for what he has said is an impossibility. He has evidently altogether mistaken the problem. Mr. Hopkins's method stands unimpaired by his criticisms. Indeed Mr. Hopkins was not a man to advance a theory which could be apparently set aside by such slender means.

JOHN H. PRATT

\section{A THEORY OF A NERVOUS ATMOSPHERE}

UNDER the above title, Dr. Richardson, in a lecture published in the Medical Times and Gazette of last week, suggests a new theory in respect to nervous function. We propose in a few sentences to state simply the meaning of this theory.

The earlier physiological writers on the functions of the nervous system were under the impression that the brain, spinal cord, and other nervous centres acted after the manner of glands, and produced or secreted, as they said, a liquid. They called this assumed secreted liquid the nervous fluid, and they considered that it charges the nervous system, some also supposing that it makes even a circulation through tubular nervous channels or canals. It was not an uncommon notion that the nervous fluid conveys nourishment to the organs of the body; but the most common, and indeed generally accepted, hypothesis was, that it acts as a means of communication between all parts of the nervous system, and is the communicating medium of the impressions and motions derived from the outer world. Attempts were made to measure the rate of motion through this fuid, how long it took to convey an impression by it from brain to muscle.

The discovery of frictional electricity, the special discovery of the electric shock by Cuneus, of Leyden, in 1746, and the after discovery by Galvani of the inductive action of the prime conductor of the electrical machine on the muscles of frogs, threw quickly into the shade the speculations of the earlier neuro-physiologists. It was assumed at once that there exists a true animal electricity, that there is production of electricalaction within the bodies of all living animals, that there is conduction, and, in short, every mechanism and method for the carrying on, if we may so say, of electrical life. The discovery of the electrical organs of the torpedo, the dissection of the animal, the descriptions of its nerves by John Hunter, and the experiments made by a very earnest investigator, Mr. Walsh, aided greatly to establish the hypothesis which Galvani and his followers advanced, and which Volta, with the whole force of his experimental argument, failed to demolish.
Of late years the old hypothesis of the nervous fluid has been lost altogether, while the electrical hypotresis infinitely varied from its original and simple character, and infinitely varying with every new step of electrical discovery, has in a certsin sense retained its popular hold. It is true the hypothesis has rested on so much laboured obscurity that nobody has succeeded in making out of it a demonstration like the demonstration of the circulation of the blood, and no one has made it so simple that every scholar can read it when it is written, and every medical practitioner practise by it and act upon it as a known principle. It is true that since the time when Volta gave his undeniable proofs against the truth of the first inferences of Galvani, the best and most thoughtíl philosophers have felt doubts as to the electrical character of living action, and have looked on Galvani's construction of life as a beautiful crumbling ruin rather than as a temple befitting the worship of the gods of nature; and, lastly, it is true that whoever takes up to read the tomes or volumes of the most eminent writers on the subject of animal electricity is prone to lay them down again as he would the handles of a battery that master his will without appealing to his reason. All this is quite true; but still the electrical hypothesis has, as we before said, held its place; no attempt has been made to replace it; it has maintained around it a spell of fascination.

The theory that has been suggested by Dr. Richardson is in some sense a retum to the old view respecting nervous action, and in some sense also is an exterision to the nervous system of the physical idea of communication of motion by molecular disturbance. In a few words, the author of the theory supposes that the blood, as it circuIates in the vessels on which the structures of the body are constiucted, yields a diffusible vapour or atmosphere which charges the nervous system surrounding the molectules of nervous matter and pervading the whole nervous organism. He attempts to formulate the physical qualities of this vapour; it is probably an organic vapour containing carbon, hydrogen, and nitrogen; it is insoluble in blood, it is condensible by cold, diffusible by heat; it is retained after death longer in cold-blooded animals than in warm-blooded, and longer in warm-blooded animals that have died in cold than in those that have died in heat; it possesses conducting power, and as a physical substance is susceptible of variation of pressure; it connects the nervous system in all its parts together; it is the medium of communication during life between the outer and the inner existence; by the organs of the senses the impressions and motions derived from the outer worlcl are vibrated into or through the nervous atmosphere to the brain; in the living and healthy animal the nervous ether, if we may so designate it, is in correct tension, in the feeble it is diminished, in the dead it is absent or inactive; in the waking times of the living it is most active; it may be used up faster than it is produced during exercise; it is renewed during sleep.

On the supposition of the existence of a nervous ether or atmosphere as thus suggested, the author of the theory accounts for various phenomena connected with the partial or complete destruction of conscious, and even of organic life. The action of narcotic vapours is an illustration in point. It is assumed that these vapoursvapours of chloroform or alcohol, for example-taken into the blood and carried to the nervous system, become diffused through the nervous atmosphere, and by their presence interfere with its physical qualities and thus obscure function. "The foreign vapour that has been introduced benumbs; in other words, it interferes with the physical conduction of impressions through what should be the cloudless atmosphere between the outer and the inner existence."

Carrying out in a different way the same line of thought, the author of the theory to which we have 